\title{
Virtual subpixel approach for single-mask phase-contrast imaging using Timepix3
}

Dreier, E.S.; Silvestre, C.; Kehres, J.; Turecek, D.; Khalil, M.; Hemmingsen, J.H.; Hansen, O.; Jakubek, J.; Feidenhans'I, R.; OIsen, U.L.

Published in:

Journal of Instrumentation

Link to article, DOI:

10.1088/1748-0221/14/01/C01011

Publication date:

2019

Document Version

Peer reviewed version

Link back to DTU Orbit

Citation (APA):

Dreier, E. S., Silvestre, C., Kehres, J., Turecek, D., Khalil, M., Hemmingsen, J. H., Hansen, O., Jakubek, J., Feidenhans'I, R., \& Olsen, U. L. (2019). Virtual subpixel approach for single-mask phase-contrast imaging using Timepix3. Journal of Instrumentation, 14(01), [C01011]. https://doi.org/10.1088/1748-0221/14/01/C01011

\section{General rights}

Copyright and moral rights for the publications made accessible in the public portal are retained by the authors and/or other copyright owners and it is a condition of accessing publications that users recognise and abide by the legal requirements associated with these rights.

- Users may download and print one copy of any publication from the public portal for the purpose of private study or research.

- You may not further distribute the material or use it for any profit-making activity or commercial gain

- You may freely distribute the URL identifying the publication in the public portal 
Prepared FOR SUbMission to JINST

$20^{\text {TH }}$ IWoRiD Workshop on Radiation Imaging Detectors

SUNDSVALL

2018

\section{Virtual Subpixel Approach for Single-Mask Phase-contrast Imaging Using Timepix3}

Erik Schou Dreier, ${ }^{a, 1}$ Chantal Silvestre ${ }^{b, c}$ Jan Kehres ${ }^{d}$ Daniel Turecek ${ }^{e, f}$ Mohamad Khalil $^{d}$ Jens H. Hemmingsen ${ }^{b}$ Ole Hansen ${ }^{b}$ Jan Jakubek ${ }^{e}{\text { Robert Feidenhans' }{ }^{g} \text { Ulrik Lund Olsen }}^{d}$

${ }^{a}$ Niels Bohr Institute, University of Copenhagen,

Universitetsparken 5, 2100, Copenhagen, Denmark

${ }^{b}$ Department of Micro- and Nanotechnology, Technical University of Denmark, Ørsteds Plads - Bygn. 347, 2800, Lyngby, Denmark

${ }^{c}$ DTU Danchip, Technical University of Denmark, Ørsteds Plads - Bygn. 345C, 2800, Lyngby, Denmark

${ }^{d}$ Department of Physics, Technical University of Denmark,

Fysikvej, 2800, Lyngby, Denmark

${ }^{e}$ Advacam s.r.o.,

U Pergamenky 1145/12, Prague 7, Prague, 17000, Czech Republic

${ }^{f}$ Center for Advanced Preclinical Imaging (CAPI), First Faculty of Medicine, Salmovska 3, Praha, 120 00, Czech Republic

${ }^{g}$ European XFEL GmbH,

Holzkoppel 4, 22869, Schenefeld, Germany

E-mail: erik.dreier@nbi.ku.dk

\footnotetext{
${ }^{1}$ Corresponding author.
} 
Aвstract: X-ray phase contrast imaging provides a method to distinguish materials with similar density and effective atomic number, which otherwise would be difficult using conventional X-ray absorption contrast. In recent years, multiple methods have been developed to acquire X-ray phase contrast images using incoherent laboratory sources. The single mask edge illumination setup has been demonstrated as a possible candidate for large scale applications due to its relaxed restrictions on longitudinal coherence and mask alignment, and for its ability to do bi-directional phase contrast images in a single sample exposure. Unfortunately, the single mask edge illumination setup's refraction sensitivity, and thereby signal to noise, is limited by detector artifacts. Furthermore, it requires multiple exposures to perform dark-field imaging, a method that enables imaging of micro-structures smaller than the image resolution.

We propose using an Advapix detector with Timepix3 pixel-readout chip in a single mask imaging setup to improve signal to noise ratio in phase contrast images. This is achieved using the Timepix 3 chip's ability to simultaneously acquire fast time of arrival and time over threshold measurement of single photon events, which enables sub-pixel identification of individual photons. In this paper, we demonstrate that signal to noise ratio can be improved by at least $67 \pm 5 \%$ using subpixel identification of single photons compared to conventional acquisitions methods. Thereby the required sample dose can be reduced considerably. This shows that there is a great potential in using Timepix 3 chip to improve $x$-ray phase contrast imaging. Further, the results indicate the possibility for dark field imaging in a single sample exposure using Timepix 3 in a single mask edge illumination setup.

KeYwords: X-ray detectors, Inspection with x-rays, X-ray radiography and digital radiography (DR), Data processing methods. 


\section{Contents}

1 Introduction 1

2 Single Mask Edge Illumination 2

3 Subpixel Positioning with Timepix3 Chip 3

4 Discussion and Outlook $\quad 7$

$\begin{array}{llr}5 & \text { Acknowledgment } & 8\end{array}$

\section{Introduction}

X-ray Phase Contrast (XPC) imaging has drawn much attention for its ability to image biological samples with a considerably lower sample dose than conventional X-ray absorption imaging. This is achieved by using X-ray refraction as image contrast, which in particular gives a high sensitivity for light materials with low effective atomic number. Multiple techniques have been developed over the last two decades to implement XPC imaging for laboratory sources [1-6]. All of these techniques are multi-modal, making it possible to acquire absorption and XPC images simultaneously as well as dark field images of objects smaller than the image resolution [7-9].

The Double Mask Edge illumination (DM-EI) XPC imaging technique[2] has already been applied for large scale applications [10]. The technique's major advantages are its eased restriction on mask alignment, low sample dose, and ability to use the entire energy spectrum of a conventional laboratory source[11]. DM-EI is a non-interferometric technique that uses a pre-sample mask to create beamlets and a detector mask to analyze the beamlet's position. By scanning the detector mask, the beamlets' intensity distributions are obtained. A minimum of two steps is required to detect a phase shift of the beamlets due to a sample and three steps to get a dark field image[9]. DMEI is commonly only implemented with refraction sensitivity along one direction as two-directional sensitivity requires complicated masks and at least three steps of the detector mask[12].

To overcome the above problems of the DM-EI setup, single mask techniques have been developed. Here, the detector mask is removed and the beamlets' position are analyzed with the detector directly. Two approaches exist: The beam-tracking technique[5], where the detector pixels are sufficiently smaller than the beamlet, and the Single Mask Edge Illumination (SMEI) technique, where the pixel borders constitute the analyzing edges of the setup[3]. The latter shows promising result for XPC imaging when compared to the DM-EI setup. The setup has high refraction sensitivity, as a small movement of the beamlet causes a large shift in intensity between the illuminated pixels, and two-directional sensitivity can be obtained in a single sample exposure using a two-dimensional pre-sample mask[13]. Unfortunately, its performance is limited by charge sharing in the detector[14], and it is not possible to obtain dark field images in a single exposure. 

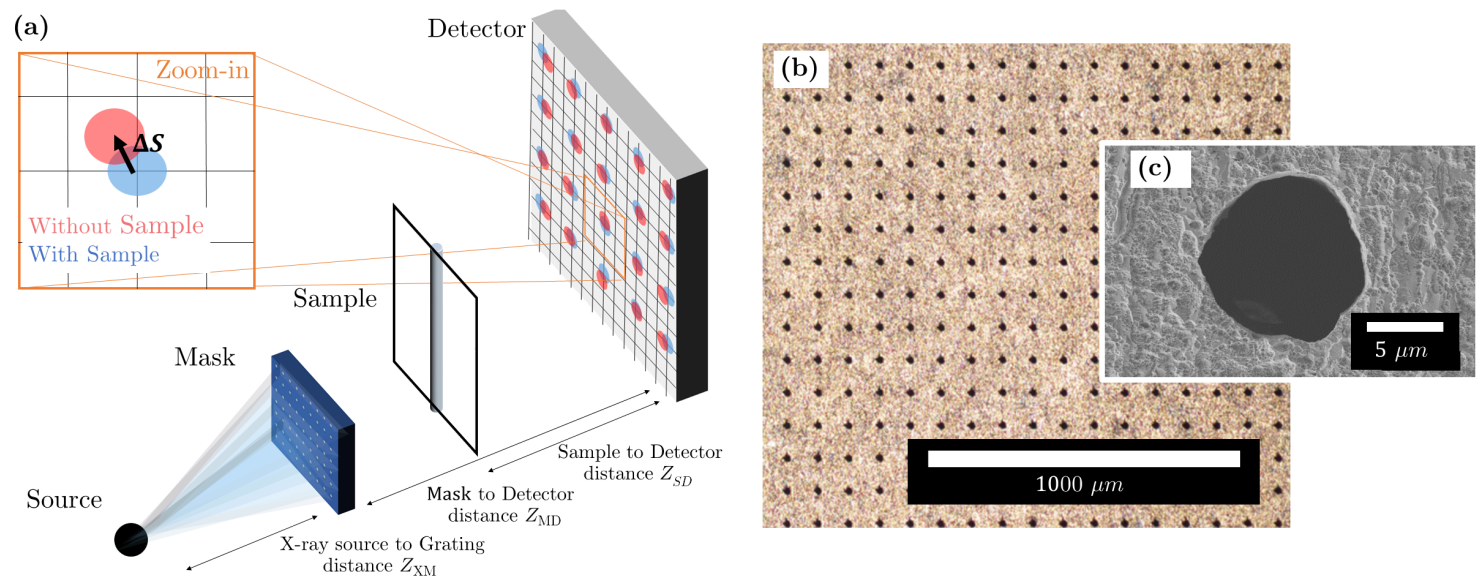

Figure 1. Illustration of the SM-EI setup at DTU (a). Optical image of tungsten mask (b) with electron microscope image showing a single hole (c).

We propose that photon counting detectors with the Timepix 3 pixel-readout chip, developed by the Medipix consortium, could minimize the limitation of the SM-EI method originally developed by Krejci et al[13]. This is possible due to the Timepix 3 chip's data-driven readout architecture which enables the measurement of the excited charge distribution in the detector of each individual photon at a hit rate of $40 \mathrm{Mhits} / \mathrm{s} / \mathrm{cm}^{2}[15]$. By analyzing the measured charge distribution, it is possible to identify each photon's sub-pixel position[16], making it possible to decrease the effective pixel size. In this paper, we focus on the use of photon sub-pixel positioning to improve signal to noise ratio in XPC images acquired with a SM-EI setup.

\section{Single Mask Edge Illumination}

The SM-EI setup employed at the Technical University of Denmark (DTU) is shown in fig. 1a. The setup uses a Hamamatsu Photonics L12161-07 micro focus X-ray source, which for the experiments presented in this paper was set to a source spot size of $5 \mu \mathrm{m}, U=50 \mathrm{kV}$, and $I=67 \mu \mathrm{A}$. An absorption mask is placed $Z_{\mathrm{XM}}$ from the source. The absorption mask was made with laser ablation of a $200 \mu \mathrm{m}$ thick Tungsten (W) foil and is designed with a two-dimensional array of holes with a diameter 12.3 $\pm 0.1 \mu \mathrm{m}$ and a pitch of $100 \mu \mathrm{m}$ as illustrated in fig. 1(b-c) [17]. An Advapix detector with a Timepix 3 pixel-readout chip, $1 \mathrm{~mm}$ Silicon sensor, and $55 \mu \mathrm{m}$ pixelsize, is placed $Z_{\mathrm{MD}}$ from the mask. By adjusting $Z_{\mathrm{MD}}$, the beamlets that pass through the mask has a spacing equal to an integer number of pixels at the detector. Through translation of the detector in the two directions perpendicular to the X-ray beam, the mask is aligned with the detector such that the beamlets hit the pixel corners. The sample is placed between mask and detector at $Z_{\mathrm{SD}}$ from the detector.

The SM-EI technique allows for retrieval of a bi-directional phase images in a single shot. The XPC image is acquired by finding the shift of each beamlet

$$
\Delta \mathbf{S}=\mathbf{S}_{\text {Sample }}-\mathbf{S}_{0}
$$

where $\mathbf{S}_{\text {Sample }}$ and $\mathbf{S}_{0}$ is the beamlet's position with and without a sample respectively. The beamlet's position is found through a weighted mean of the intensity $I_{p}$ in the $M$ pixels surrounding 

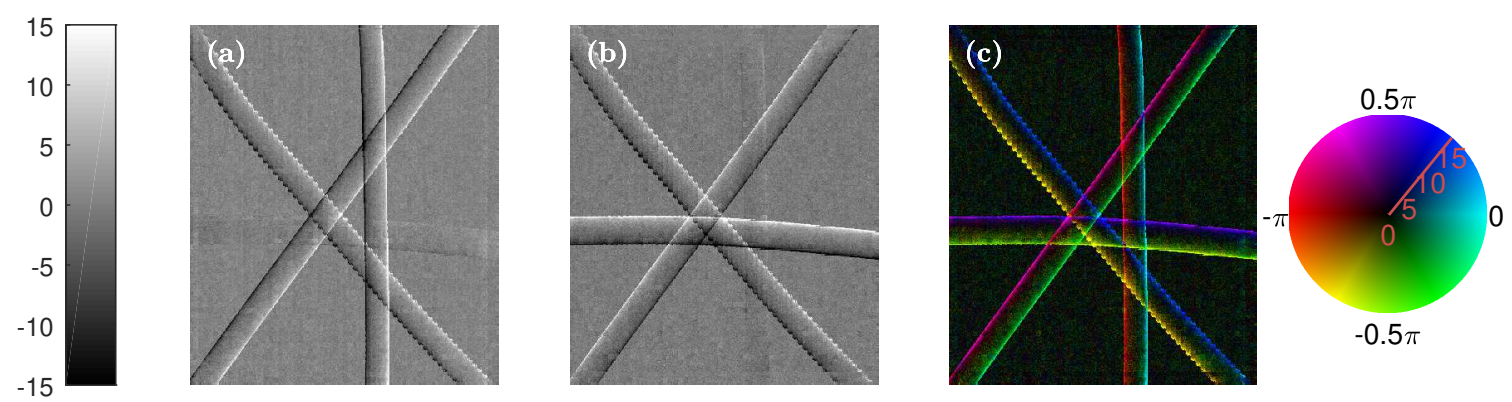

Figure 2. Bi-direction XPC image of 4 crossed nylon wires using the horizontal $\Theta_{x}$ (a) and vertical $\Theta_{y}$ (b) refraction angle of the X-ray beamlets as contrast. The color scale (left) is given in $\mu \mathrm{rad}$. The refraction signal in the two directions can can be combined in an HSV color image (c), where the color shows the direction of the refraction and brightness the amplitude of the refraction angle.

the illuminated corner

$$
\mathbf{S}=\frac{\sum_{p=1}^{M} \mathbf{P}_{p} I_{p}}{\sum_{p=1}^{M} I_{p}},
$$

where $\mathbf{P}_{p}$ is the pixel position. The refraction angle of the beamlet passing through a sample along the horizontal $x$ and vertical direction $y$ is then given by

$$
\Theta_{x}=\tan ^{-1}\left(\Delta S_{x} / Z_{\mathrm{SD}}\right) \quad \text { and } \quad \Theta_{y}=\tan ^{-1}\left(\Delta S_{y} / Z_{\mathrm{SD}}\right) .
$$

An image of 4 crossed wires acquired with the setup at DTU is shown in figure 2. The image was obtained by raster scanning the sample in $6 \times 6$ steps with a step size of $20 \mu \mathrm{m}$ to obtain higher resolution than the mask pitch. As clearly shown by the HSV color-plot in figure $2 c$, the bi-directional sensitivity allows for clear detection of all wires, which is difficult using the refraction along horizontal or vertical direction alone (figure 2a-b).

\section{Subpixel Positioning with Timepix3 Chip}

Charge sharing between detector pixels can induce a current in multiple adjacent pixel's electrodes. When the current induced in an electrode is higher than the triggering threshold, it will be registered as an event. By recording the current's Time over Threshold (ToT) in the electrode, the energy deposited in that pixel can be found [18]. The Timepix 3 chip is capable of reading both Time over Threshold (ToT) as well as the Time of Arrival (ToA) at a resolution of $1.56 \mathrm{~ns}$ for each single event. This enables the possibility to identify clusters of events belonging to the same photon interaction. The ToT measurement, and hence the amount of energy deposited in each pixel, can then be used to find the position of the photon interaction[16]

$$
\mathbf{R}_{\mathrm{Ph}}=\frac{\sum_{e=1}^{N} \mathbf{P}_{e} E_{e}}{\sum_{e=1}^{N} E_{e}},
$$

where $\mathbf{P}_{e}$ and $E_{e}$ are the pixel position and the deposited energy of the $e^{\prime}$ th event, and $N$ is the total number of events in the cluster of pixels triggered by the photon. 

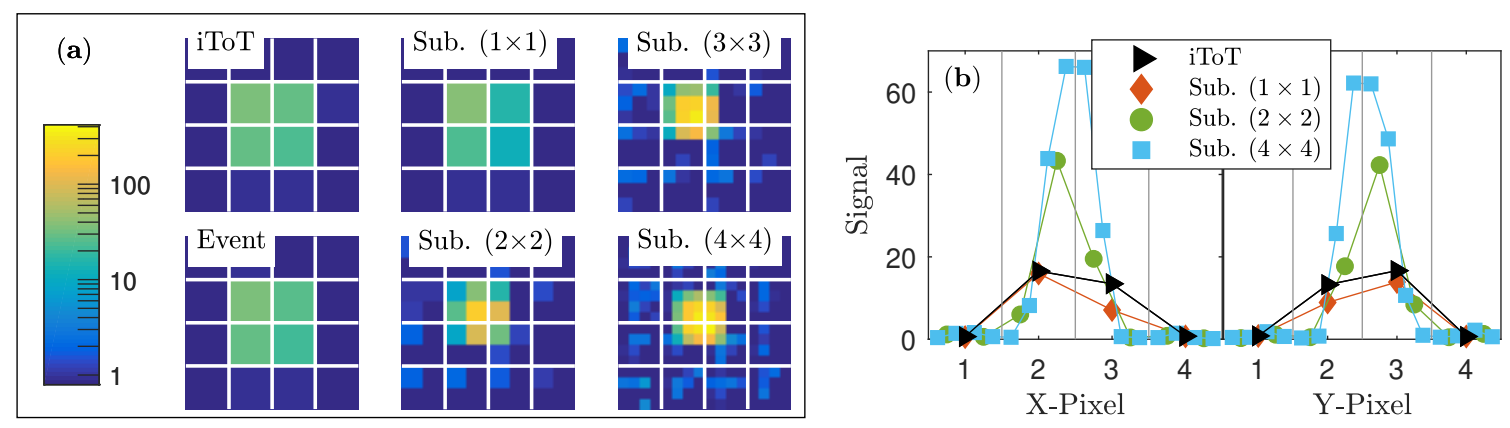

Figure 3. Image of a single beamlet acquired using different data processing methods (a), with horizontal and vertical profiles of the beamlets' distribution shown for selected methods (b). The image signal has been normalized to the standard deviation in the non illuminated pixels. The lines mark pixel borders.

Close to a pixel border, the probability of charge sharing increases and hence the number of pixels that are triggered per photon. In order to obtain a best estimate of the single photon position, a high number of triggered pixels is desired [16]. The probability of charge sharing is maximized by adjusting the position of the detector such that beamlets hit the pixel corners.

Using Advacam developed interface software Pixet, each photon's position is calculated and afterwards all photons are binned into $(n \times n)$ virtual "subpixels" per pixel. We will compare the results of subpixel rebinning the photons to the more conventional integrated ToT (iToT), where the ToT is integrated across all events in every pixel, and event count (Event), where just the number of events in each pixel is summed regardless of deposited energy. To avoid experimental variation affecting the comparison, all experiments are conducted with data being saved in a raw data format. For each event, pixel position, ToA, and ToT are recorded. After acquisition, the different processing methods are applied to the raw data. For all methods, the conversion of ToT into energy developed by Jakubek[18] has been employed. Only single photons causing clusters equal to or larger than two pixels are included for the subpixel methods. In principle, it is necessary to have at least 3 events per single photon cluster in order to determine a single photon's position in the pixel plane, however, the ensemble average of all photons' position was found to be more precise by also including two pixel clusters.

In figure 3, we show how subpixel rebinning can be used to resolve the distribution of a single beamlet hitting the corner of 4 pixels. For this measurement, we used $Z_{\mathrm{MD}}=513 \mathrm{~mm}$ and $Z_{\mathrm{SD}}=315 \mathrm{~mm}$. Including only the geometric expansion of the beamlets due to the fan-beam setup and assuming a gaussian beamlet shape, this geometry would result in the beamlets having an expected FWHM at the detector of $\varnothing \sim 27 \mu \mathrm{m}$. As seen in figure 3, using subpixel identification of each photon increases the Signal to Noise Ratio (SNR).

To evaluate the accuracy and precision of the beamlet's position for different data processing methods, a single beamlet was moved across the detector. The beamlet was made with a pinhole mask with similar holesize as the 2D mask. The mask was scanned, moving the beamlet across the detector in $10 \times 20$ points in horizontal and vertical direction, respectively, with a spacing of $20 \mu \mathrm{m}$ between the points in both directions. The detector was tilted by $\sim 1^{\circ}$ as compared to the movement the beamlet scan. When projecting the scan into a single pixel, the tilt ensures that the entire area of the pixel is covered with a spacing of $2-5 \mu \mathrm{m}$ between the beamlet's center positions. 

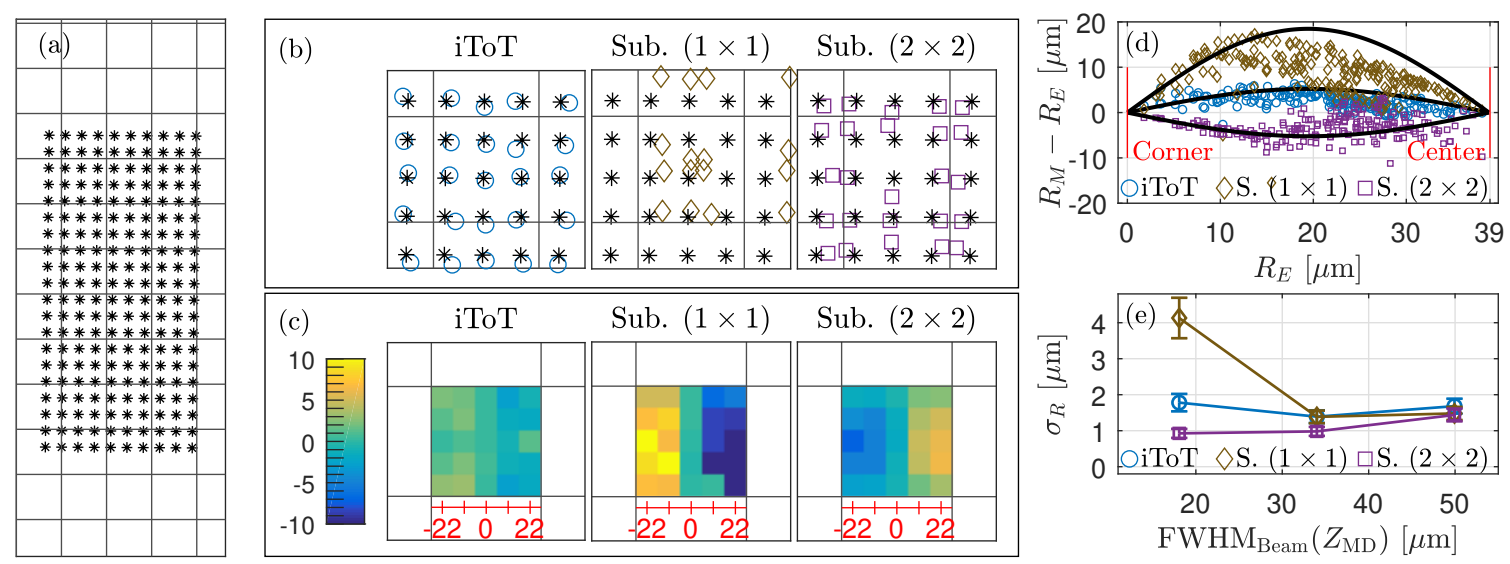

Figure 4. Comparison of expected $\mathbf{S}_{E}$ and measured $\mathbf{S}_{M}$ position using different readout methods as the beamlet is scanned across the detector. The scan covers $10 \times 20$ position across the detector (a). A zoom in on $2 \times 2$ pixels (center to center) illustrates the difference between $\mathbf{S}_{E}$ (black stars) and $\mathbf{S}_{M}$ (colored symbols) for the tree methods (b). The black lines show the pixel borders. The difference in measured and expected horizontal position $\Delta S_{x}=S_{x, M}-S_{x, E}$ is shown as function of $S_{x, E}$ projected into a single pixel (c). The color scale is given in $\mu \mathrm{m}$ and the red scale bar shows the pixel position in $\mu \mathrm{m}$. The difference between measured $R_{M}$ and expected $R_{E}$ distance to the nearest corner (d) can be fitted with $F\left(R_{E}\right)$ given in eq. (3.2) (black curves) and the standard deviation $\sigma_{R}$ of the distribution of data points around $F\left(R_{E}\right)$ calculated for $R_{E}<15 \mu \mathrm{m}(\mathrm{e})$.

In figure 4 , we compare the beamlets' measured position $\mathbf{S}_{M}$, estimated by the different processing method, to the expected beamlet position $\mathbf{S}_{E}$. We determine $\mathbf{S}_{E}$ assuming a fixed beamlet spacing of $20 \mu \mathrm{m}$ and an average across all position $\overline{\mathbf{S}}_{E}=\overline{\mathbf{S}}_{\mathrm{iToT}}$, where $\overline{\mathbf{S}}_{\mathrm{iToT}}$ is the average of all the beamlet's position found with iToT data processing method. By subtracting the expected position of the beamlet from the position estimated by the different method $\mathbf{S}_{M}$, the detector response can be evaluated. As seen from figure 4c, the different ways of processing the data affect the detector response differently. The iToT seems to predict the beamlet position most accurately with smallest difference between the measured and expected position, generally tending to push the beamlet's predicted position towards the center of the pixel. It is seen clearly from both figure $4 \mathrm{~b}$ and $4 \mathrm{c}$, that rebinning into a single pixel after subpixel localizing each individual photon seems to over predict the beamlet's proximity to the pixel centers. Opposite, rebinning into a $2 \times 2$ subpixel grid, decreasing the virtual pixel size to $27.5 \mu \mathrm{m}$, seems to push the predicted position of the beamlets towards the corners.

The same behavior is clearly seen in the average distance to the nearest corner, shown in figure $4 \mathrm{~d}$. Here, the single pixel rebinning over-predicts the position of the beamlet with more than $15 \mu \mathrm{m}$ towards the beam center at a distance of $15 \mu \mathrm{m}$ from a corner. However, the figure also seems to indicate that the variation of the points is smaller for the $2 \times 2$ subpixel binned data than the others. This is quantified through fitting

$$
F\left(R_{E}\right)=A \sin \left(\frac{R_{M}-R_{E}}{2 \pi / D}\right),
$$

where $D=77.8 \mu \mathrm{m}$ is the diagonal of the pixel, and $A$ is the variable amplitude. As a measure of the precision, we calculate the standard deviation of the measured radius distribution $\sigma_{R}$ around 

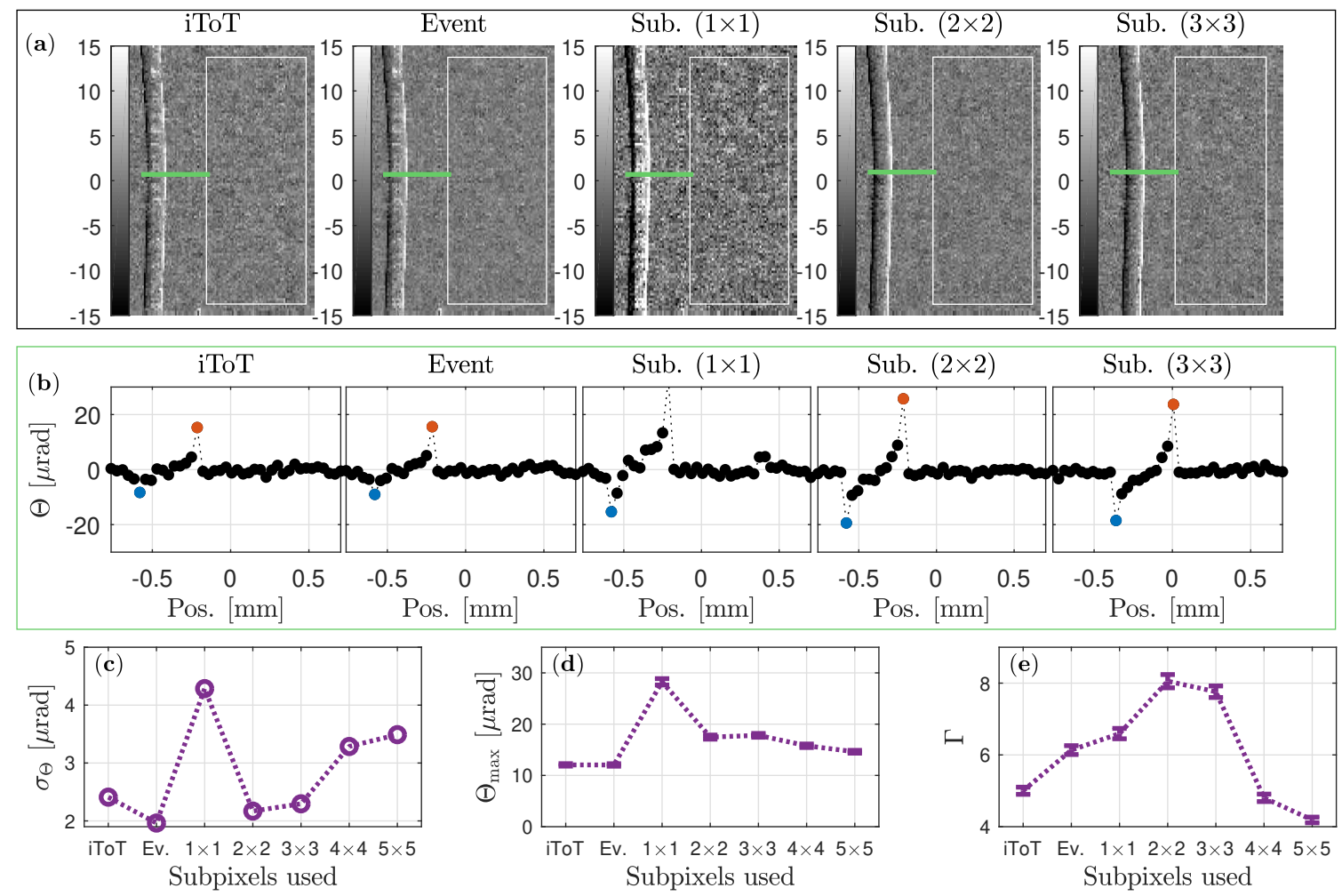

Figure 5. XPC image of a $300 \mu \mathrm{m}$ thick nylon wire. The effect of different processing methods on the XPC images of a wire (a). The green lines mark the XPC profiles of the wire shown (b) where the blue and red markers illustrate the maximum refraction signal in the two directions. For the different methods we calculate the standard deviation of the background XPC image $\sigma_{\Theta}(\mathrm{c})$, mean maximum refraction across both direction $\Theta_{\max }(\mathrm{d})$, and the signal to noise ratio $\Gamma=\Theta_{\max } / \sigma_{\Theta}(\mathrm{e})$.

$F\left(R_{E}\right)$ at $R_{E}<15 \mu \mathrm{m}$. We do so for 3 different distances of $Z_{\mathrm{MD}}$ corresponding to three different

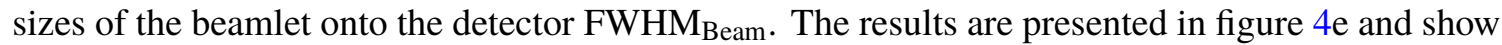
that rebinning into subpixels $(2 \times 2)$ seems to give a much more precise measure of the beamlet's position than any of the two other methods at small beamlet sizes (small $Z_{\mathrm{MD}}$ ). As FWHM Beam is increased, the precision of the beamlet's position is decreased. When FWHM Beam $_{\text {approaches the }}$ size of the pixel, the effect of using subpixel positioning disappears.

The above implies that a more precise positioning of the beamlet can be achieved using the subpixel methods. To evaluate the effect of this on the XPC sensitivity, a $300 \mu \mathrm{m}$ thick nylon wire was measured at $Z_{\mathrm{SD}}=190 \mathrm{~mm}, Z_{\mathrm{MD}}=358 \mathrm{~mm}$, and $Z_{\mathrm{XM}}=289 \mathrm{~mm}$. At this geometry, the beamlets were hitting every fourth pixel corner. The phase shift along the entire profile of the wire was measured through raster scanning the sample in $7 \times 2$ steps along the horizontal (perpendicular to the wire) and vertical direction respectively. The exposure time was set to $15 \mathrm{~s}$ for each measurement point. The results of this experiment is shown in figure 5. It is clearly seen from the XPC images and profiles of the wire in figure 5(a-b), that the phase shift prediction is largely affected by the accuracy of the beamlet's position. Most clearly seen from the different prediction of peak refraction, where the large inaccuracy of the $(1 \times 1)$ subpixel results in a much larger prediction of refraction angle. Similarly, there is a discrepancy between the other data processing methods. 
To quantify this, we calculate the standard deviation of the background signal $\sigma_{\Theta}$ within the white rectangle in figure 5a and the average maximum absolute refraction $\Theta_{\max }$ along the whole wire. The result can be seen in figure 5(c-d), where the over-prediction of refraction from $(1 \times 1)$ subpixel rebinning is clearly seen in both $\Theta_{\max }$ and $\sigma_{\Theta}$ values. Furthermore, it is generally observed that the refraction angle is predicted differently for each method.

To compare the different methods, we use the ratio $\Gamma=\Theta_{\max } / \sigma_{\Theta}$ as a measure of signal to noise ratio in the images. The result is plotted in figure $5 \mathrm{e}$ and shows that $\Gamma$ is maximized when using $(2 \times 2)$ subpixels. The difference between iToT and $(2 \times 2)$ subpixel is $67 \pm 5 \%$. It is noted, that increasing the distance $Z_{\mathrm{MD}}$ so that the beamlet hit every 5 th, 6th, or 7 th pixel decreases the gain from using subpixel to below $20 \%$.

\section{Discussion and Outlook}

We have in this paper demonstrated that using Timepix 3 chip for SM-EI XPC imaging could improve the technique's sensitivity significantly. In figure 5 , it is seen in the peak refraction angle to background noise ratio $\Gamma$ increases with $67 \pm 5 \%$ when using $(2 \times 2)$ subpixel compared to the normal charge integrating iToT. However, the result was found largely dependent on the setup's geometry. Assuming that $\Gamma$ is proportional to the square root of the acquisition time, the $(2 \times 2)$ subpixel method could reduce the sample exposure time to $36 \%$ of what is needed for the iToT method.

It was shown in figure 4 that subpixel data processing methods greatly reduced the accuracy of the beamlet's measured position through a systematic shift compared to the expected. We suspect that it is the systematic inaccuracy that affects the measured $\sigma_{\Theta}$ and $\Theta_{\max }$ in figure 5. Similarly, data processing artifacts are likely causing the substantial decrease in $\Gamma$ when using more than $(4 \times 4)$ rebinning in figure 5. The tendency of inaccurate measurements of beamlet position should be correctable with models of data processing artifacts. If these inaccuracies were properly accounted for, we expect $\Theta_{\max }$ to be the same for all methods, and the difference between the methods to be seen solely in $\sigma_{\Theta}$.

The sensitivity of the XPC imaging setup is determined by the measurement precision of the refraction angle of each beamlet, which is controlled by the precision of the change in beamlet position on the detector.

Figure 4 shows that the precision is significantly better when using $(2 \times 2)$ subpixel positioning for the beamlet placed close to a corner as compared to iToT method. We demonstrated that the best precision of the beamlet's position was $0.9 \pm 0.1 \mu \mathrm{m}$ using $(2 \times 2)$ subpixel method. For this experiments, the mask was translated with Newport IMS500CC translation stages in both direction. The accuracy of the motors, measured as the minimum to maximum deviation of the desired position (not standard deviation) across the full travel length $(500 \mathrm{~mm})$ is expected to be 3 $\mu \mathrm{m}$ for both directions. Furthermore, the motor has an expected 1 standard deviation bi-directional reproducibility of $0.5 \mu \mathrm{m}$. Therefore, the precision of $0.9 \pm 0.1 \mu \mathrm{m}$ is most likely dominated by imprecise motor translation. 
In comparison, the precision of the beamlets' measured movement in the XPC images in figure 5 is significantly better than the above. $\sigma_{\Theta}$ in the XPC image was for iToT found to be $\sigma_{\Theta, \text {, ТоT }}=2.4$ $\mu \mathrm{rad}$, which corresponds to an uncertainty on the beamlet's measured position of $\sigma_{\Delta S_{x} \text {, iToT }}=0.49$ $\mu \mathrm{m}$ on the detector.

The expected error on the mean of the actual beamlet position due to statistical variation is given by $\sigma_{S_{x}}=\frac{\operatorname{FWHM}_{\mathrm{Beam}}(2 \sqrt{2 \ln (2)})^{-1}}{\sqrt{(} N)}$, assuming a Gaussian beam distribution. $N \sim 3000$ is the number of photons in each beamlet, found from the number of excited clusters per beamlet. At $Z_{\mathrm{MD}}=358 \mathrm{~mm}$, we expect $\mathrm{FWHM}_{\mathrm{Beam}}=27 \mu \mathrm{m}$, which results in $\sigma_{E, S_{x}}=0.21 \mu \mathrm{m}$. Assuming a low absorbing sample (the same number of photons with and without a sample) this gives an uncertainty on the beam positioning of $\sigma_{\Delta S_{x}, E}=\sqrt{2} \sigma_{S_{x}, E}=0.30 \mu \mathrm{m}$. Assuming that the entire change in $\Gamma$ is due to a change in uncertainty of the measured refraction, $\sigma_{\Theta}$ could decrease with as much as $67 \%$ when using the $(2 \times 2)$ subpixel method, making $\sigma_{\Delta S_{x},(2 \times 2)} \sim 0.3 \mu \mathrm{m} . \sigma_{\Theta}$ found in this experiment, therefore, seems to be at the limit of statistical variation in the actual beamlet position. The actual beamlet position is controlled by the aperture size of the mask, the source size, $Z_{\mathrm{MD}}, Z_{\mathrm{SD}}$, and the number of photons through each hole (the product of photon flux and count time). If the beamlet size is the dominating contributor to $\sigma_{\Theta}$, decreasing the aperture size would increase the XPC sensitivity as function of sample exposure. However, more experiments are required to determine the lower limit of the angular resolution of the SM-EI technique using subpixel methods.

The SM-EI XPC setup presented in this paper shows great potential for further development using the increased information obtained with subpixel positioning of the photon. Firstly, the present setup uses a mask with $100 \mu \mathrm{m}$ pitch, and hence it was necessary to raster scan the sample to obtain information of the entire sample as done for the nylon threads in figure 2. Using subpixel positioning, it should be possible to acquire XPC images even if the beamlets hit every corner as

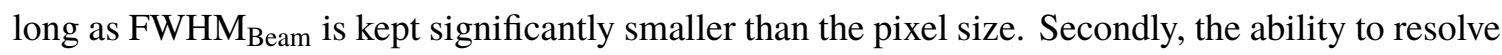
the beamlet's shape with multiple points as shown in figure 3, should enable dark field imaging as demonstrated with the beam tracking techniques (using a single mask and a high resolution detector)[5]. Future work will investigate the possibility to use the Timepix 3 chip to acquire dark field images in a single sample exposure.

\section{Acknowledgment}

The authors acknowledge Innovation Fund Denmark and the EUROSTARS programs for financing their research work, and acknowledge the MEDIPIX collaborations who are responsible for the development of the Timepix 3 ASIC used in this work.

\section{References}

[1] F. Pfeiffer, T. Weitkamp, O. Bunk, and C. David. Phase retrieval and differential phase-contrast imaging with low-brilliance X-ray sources. Nature Physics, 2:258-261, 2006.

[2] A. Olivo and R. Speller. A coded-aperture technique allowing X-ray phase contrast imaging with conventional sources. Applied Physics Letters, 91(074106), 2007. 
[3] F. Krejci, J. Jakubek, and M. Kroupa. Hard x-ray phase contrast imaging using single absorption grating and hybrid semiconductor pixel detector. Review of Scientific Instruments, 81(113702), 2010.

[4] K. S. Morgan, D. M. Paganin, and K. K. W. Siu. Quantitative single-exposure x-ray phase contrast imaging using a single attenuation grid. Optics Express, 19(20), 2011.

[5] F. A. Vittoria, M. Endrizzi, P. C. Diemoz, A. Zamir, U. H. Wagner, C. Rau, I. K. Robinson, and A. Olivo. X-ray absorption, phase and dark-field tomography through a beam tracking approach. Nature Scientific Reports, 5(16318), 2015.

[6] H. Wang, Y. Kashyap, and K. Sawhney. From synchrotron radiation to lab source: advanced speckle-based X-ray imaging using abrasive paper. Scientific Reports, 6(20476), 2016.

[7] F. Pfeiffer, M. Bech, O. Bunk, P. Kraft, E. F. Eikenberry, CH. Bronnimann, C. Grunzweig, and C. David. Hard-X-ray dark-field imaging using a grating interferometer. Nature Materials, 7:134-137, 2008.

[8] M. Bech, A. Tapfer, A. Velroyen, A. Yaroshenko, B. Pauwels, J. Hostens, P. Bruyndonckx, A. Sasov, and F. Pfeiffer. In-vivo dark-field and phase-contrast X-ray imaging. Scientic Reports, 3(3209), 2013.

[9] M. Endrizzi, P. C. Diemoz, T. P. Millard, J. L. Jones, R. D. Speller, I. K. Robinson, and A. Olivo. Hard X-ray dark-field imaging with incoherent sample illumination. Applied Physics Letters, 104 (024106), 2014.

[10] A. Astolfo, M. Endrizzi, F. A. Vittoria, P. C. Diemoz, B. Price, I. Haig, and A. Olivo. Large field of view, fast and low dose multimodal phase-contrast imaging at high X-ray energy. Scientific Reports, 7 (1), 2017.

[11] P. C. Diemoz, C. K. Hagen, M. Endrizzi, and A. Olivo. Sensitivity of laboratory based implementations of edge illumination X-ray phase-contrast imaging. Applied Physics Letters, 103(24), 2013.

[12] G. K. Kallon, M. Wesolowski, F. A. Vittoria, M. Endrizzi, D. Basta, T. P. Millard, P. C. Diemoz, and A. Olivo. A laboratory based edge-illumination X-ray phase-contrast imaging setup with two-directional sensitivity. Applied Physics Letters, 107(204105), 2015.

[13] F. Krejci, J. Jakubek, and M. Kroupa. Single grating method for low dose 1-d and 2-d phase contrast X-ray imaging. Journal of Instrumentation, 6(01):C01073, 2011.

[14] G K Kallon, P C Diemoz, F A Vittoria, D Basta, M Endrizzi, and A Olivo. Comparing signal intensity and refraction sensitivity of double and single mask edge illumination lab-based x-ray phase contrast imaging set-ups. Journal of Physics D: Applied Physics, 50(415401), 2017.

[15] T. Poikela, J. Plosila, T. Westerlund, M. Campbell, M. De Gaspari, X. Llopart, V. Gromov, R. Kluit, M. van Beuzekom, F. Zappon, V. Zivkovic, C. Brezina, K. Desch, Y. Fu, and A. Kruth. Timepix3: a $65 \mathrm{k}$ channel hybrid pixel readout chip with simultaneous toa/tot and sparse readout. Journal of Instrumentation, 9(05), 2014.

[16] M. Khalil, E. S. Dreier, J. Kehres, J. Jakubek, and U. L. Olsen. Subpixel resolution in cdte timepix3 pixel detectors. Submitted to Journal of Synchrotron Radiation, 2018.

[17] C. Silvestre, J. H. Hemmingsen, E. S. Dreier, J. Kehres, and O. Hansen. Microfabrication of high aspect ratio X-ray gratings using tungsten laser ablation. In preperation for MNE 2018 proceedings, 2018.

[18] J. Jakubek. Semiconductor pixel detectors and their applications in life sciences. Journal of Instrumentation, 4(03):P03013, 2009. 